\title{
Analysis of several ferroelectric materials
}

\author{
Donghui Wang \\ North China Electric Power University, Baoding, 071000, China
}

\begin{abstract}
Nowadays, ferroelectricity materials are widely used. Many researchers have put effort into it to make further exploratory. This template explains and demonstrates several ferroelectricity materials. It includes piezoelectric materials, $\mathrm{BaTiO} 3$ ceramics and multi-material iron. We take their nature and inside structure as examples to explain how they work and why. Depending on that, we can take further cognition. By my analysis, we can get a whole understanding about the ferroelectricity materials I mention below. After all, we can't get the real intrinsic nature. So ferroelectric material still has high research value. It is worth studying further.
\end{abstract}

KEYWORD: Ferroelectricity, piezoelectric, material.

\section{INTRODUCTION}

Ferroelectricity is a property of certain materials that have a spontaneous electric polarization that can be reversed by the application of an external electric field. The term is used in analogy to ferromagnetism, in which a material exhibits a permanent magnetic moment. Ferromagnetism was already known when ferroelectricity was discovered in 1920 in Rochelle salt by Valasek. Thus, the prefix ferro, meaning iron, was used to describe the property despite the fact that most ferroelectric materials do not contain iron [1].

In the text, I will list below some of the ferroelectric material: piezoelectric materials, $\mathrm{BaTiO}_{3}$ ceramics and multi-material iron. In terms of the nature and structure of these materials, I will describe these ferroelectric materials. Also I will evaluate the advantages and disadvantages of the ferroelectric materials, together with the application. So we can have a grasp with the ferroelectric material on the whole.

\section{MATERIAL INTRODUCTION}

\subsection{Piezoelectric materials.}

All ferroelectric materials have ferroelectric and piezoelectric properties [2]. Ferroelectricity means that the material will produce spontaneous polarization in a certain temperature range. Since the ferroelectric crystal lattice of positive and negative charge centers do not coincide.It can produce electric dipole moment even without external electric field. And it can change the direction of the spontaneous polarization at external electric field. When the temperature is above a critical value, its lattice structure is changed. Positive and negative charge centers coincide .And the spontaneous polarization disappears. We call this the critical temperature is Curie temperature (Tc). Fig. 1 gives an example of Tetragonal unit cell of lead titanate to show how TC works.

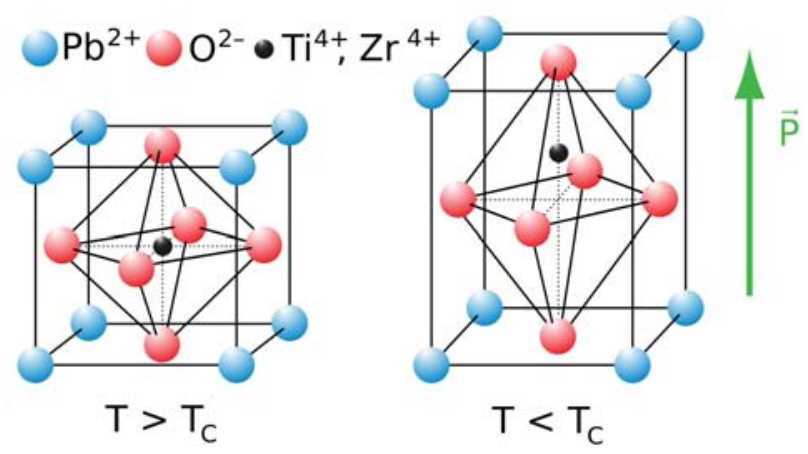

Fig. 1 Tetragonal unit cell of lead titanate

Piezoelectricity is a property of achieving mechanical energy - energy conversion .If apply force to the material in a certain direction with the materia deformed, the internal polarization occurs and it produces charge in the surface .This is the piezoelectric effect. In contrast, if the electric field is applied to the material and the material will be deformed to produce mechanical force then. This is the converse 
piezoelectric effect. Fig. 2shows the basic characteristics of the piezoelectric material.

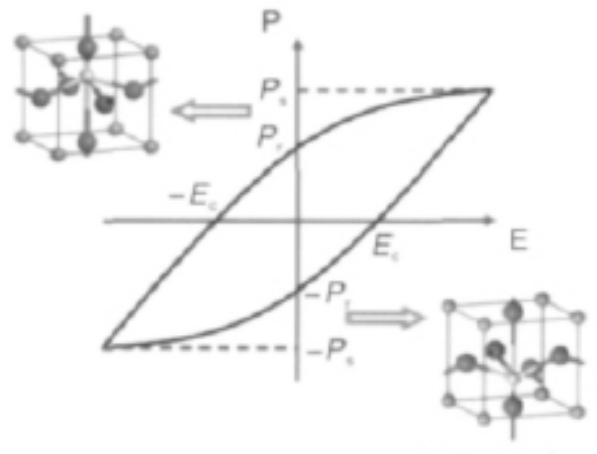

Fig. 2 Basic characteristics of the piezoelectric material

All ferroelectric materials have the above two kinds of properties. This is one of the basis to build the of electro-mechanical materials systems. With the gradual improvement of the device miniaturization requirements, Conventional piezoelectric block is gradually transition to the piezoelectric film. Especially the emergence of micro-electromechanical systems'(Micro Electromechanical System MEMS) together with the perfection of the film growth techniques make the film become a major research.

\subsection{BaTiO3 ceramics.}

Ferroelectrics which is represented by $\mathrm{BaTiO}_{3} \mathrm{Ce}-$ ramics [3] have a high dielectric constant. It is a capacitor material to manufacture ferroelectric ceramic. It is also one of the most widely used electronic ceramic materials domestic all over the world. In the case of the dielectric layer thickness determined, the higher the dielectric constant of the material is, the greater the capacitance of the capacitor is. And it is easier to make devices miniaturization. Many studies show, doping can improve the dielectric properties of $\mathrm{BaTiO}_{3}$ Ceramics. Thus it is more conducive to the storage capacitor applications. Ions may be doped with are below: $\mathrm{Nd}^{3+}, \mathrm{Ca}^{2+}, \mathrm{Sr}^{2+}, \mathrm{La}^{3+}, \mathrm{Sn}^{4}$ ${ }^{+}, \mathrm{Zr}^{4+}, \mathrm{Mg}^{2+}, \mathrm{Co}^{3+}, \mathrm{Nb}^{5+}, \mathrm{Mn}^{4+}$. Table 1 is the properties of different kinds of $\mathrm{BaTiO}_{3}$ ferroelectric ceramics.

Table 1 The properties of different kinds of $\mathrm{BaTiO} 3$ ferroelectric ceramics

\begin{tabular}{cccc}
\hline Materials & Dopings & $\varepsilon r$ & $\tan \delta / 10-4$ \\
\hline BaTiO3 & Fe2O3,ZnO & 1500 & 100 \\
BaTiO3- & MnCO3,ZnO & 20000 & 11000 \\
CaSnO3 & & & \\
BaTiO3- & & 2400 & 130 \\
Bi2(SnO3) & Nb2O5,ZnO & & \\
\hline
\end{tabular}

\subsection{Multiferroic material.}

Multiferroic material [4] refers to a material which has two or more kinds of primary iron body features. Such properties include ferroelectric, antiferroelectric, ferromagnetic and antiferromagnetic etc. Multiferroic material study is a new field of materials science and condensed matter physics. It contains a wealth of materials science and physics research as well as can expected broad application prospects. So it is necessary to make further explore.

It is quite rare to have both ferroelectric and ferromagnetic materials. This is mainly because that most of the ferroelectric performance is by the formation of the cationic center and the deviation from the lattice atoms has no d orbital electron to fill. But most magnetic formation requires a partially $\mathrm{d}$ orbital filled with electron. These two formation mechanisms are very different.

Up to date research for ferroelectric and magnetic are focused on the physical state of cohesion and related materials category. But there are a lot of problems in the basis physics applications. The main issues of concern have two aspects: First, it is the material that synthesis electric dipole with magnetic spin order coexist. Second, it is truly important to fully understand the coupling efficiency between magnetic and electrical properties in multiferroic material. This is the basis of multi-iron. So it is necessary to make further research. Fig. 3 shows the regulatory mechanism of multiferroic material between the ferroelectricity and ferromagnetism.

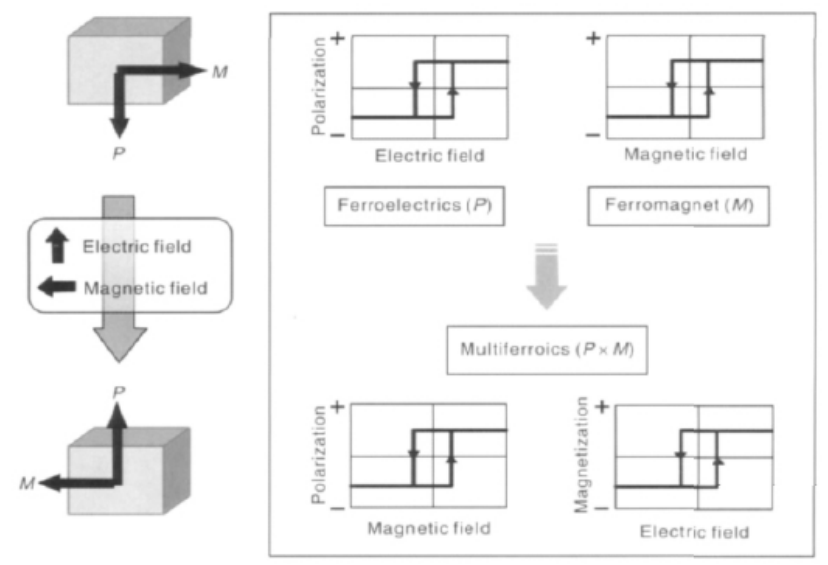

Fig. 3 Regulatory mechanism of multiferroic material between the ferroelectricity and ferromagnetism 


\section{MATERIALS ANALYSIS}

In recent years, various properties of the ferroelectric material have a lot of progress. And some have been applied to real life. But there are still many problems and difficulties about the ferroelectric material in the basic physical and technical applications. Such as ferroelectric thin films miniaturization, Semiconductor interface compatibility, resistance to fatigue and high temperature stability. Such problems are still not solved.

While the multiferroic material research also has other problems. Iron and magnetic properties of condensed matter physics research is still focused on the scope and related materials. How to synthesis of electric dipole magnetic spin order coexist material and coupling efficiency between the electric and magnetic properties are still main problems. There is still emerging ferroelectric resistive material. But it is still in early stage of development research. In short, the ferroelectric material has a broad space for development. We still need further analysis of the ferroelectric material to find the more intrinsic nature and research methods.

\section{SUMMARY}

In this text, we talk about several ferroelectricity materials. We have made some progress in their research. But the deeper things we have yet to comprehend. Ferroelectric material still has high research value.

\section{REFERENCES}

Bariumtitanate, https://en. wikipedia.org/w/index. php?title= Barium_titanate\&oldid $=724212983$

Ferroelectricity,https://en.wikipedia.org/w/index. php? title= Ferroelectricity\&oldid $=725823902$

Jiang Yin, Guoliang Yuan, Zhiguo Liu, et al. The progress of ferroelectric materials. MATERIALS CHINA. Vol. 31 (2012) No. 3, p. 26-37.

Piezoelectricity, https: //en.wikipedia.org /w/index. php ?title= Piezoelectricity\&oldid $=727068849$ 